\title{
Article \\ Sleep and Awakening Quality during COVID-19 Confinement: Complexity and Relevance for Health and Behavior
}

\author{
Teresa Paiva ${ }^{1,2,3, *}$, Cátia Reis ${ }^{1,2,4,5}$, Amélia Feliciano ${ }^{1,3,6}$, Hugo Canas-Simião ${ }^{1,3,7} \oplus^{\circ}$, Maria Augusta Machado ${ }^{8}$, \\ Tânia Gaspar 1,2,3,9, Gina Tomé 2,10, Cátia Branquinho ${ }^{2,10}$, Maria Raquel Silva ${ }^{3,11}{ }^{3}$, Lúcia Ramiro ${ }^{2,12}$,

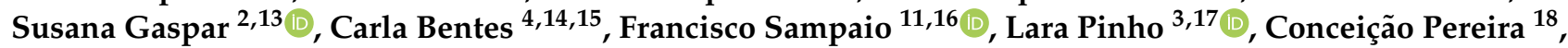 \\ Alexandra Carreiro ${ }^{19}$, Susana Moreira ${ }^{20}$, Isabel Luzeiro ${ }^{21,22,23}{ }^{\circledR}$, Joana Pimentel ${ }^{1,24}$, Gabriela Videira ${ }^{15}$, \\ Júlio Fonseca ${ }^{25}$, Ana Bernarda ${ }^{26}$, Joana Vaz Castro ${ }^{1,3}{ }^{(\mathbb{D}}$, Sofia Rebocho ${ }^{1}{ }^{(}$, Katie Almondes ${ }^{27}$, Helena Canhão ${ }^{3(\mathbb{D})}$ \\ and Margarida Gaspar Matos 2,10
}

check for

updates

Citation: Paiva, T.; Reis, C.; Feliciano, A.; Canas-Simião, H.; Machado, M.A.; Gaspar, T.; Tomé, G.; Branquinho, C.; Silva, M.R.; Ramiro, L.; et al. Sleep and Awakening Quality during COVID-19 Confinement: Complexity and Relevance for Health and Behavior. Int. J. Environ. Res. Public Health 2021, 18, 3506. https://doi.org/10.3390/ ijerph18073506

Academic Editor: Giuseppe Barbato

Received: 12 February 2021

Accepted: 16 March 2021

Published: 28 March 2021

Publisher's Note: MDPI stays neutral with regard to jurisdictional claims in published maps and institutional affiliations.

Copyright: (C) 2021 by the authors Licensee MDPI, Basel, Switzerland. This article is an open access article distributed under the terms and conditions of the Creative Commons Attribution (CC BY) license (https:// creativecommons.org/licenses/by/ $4.0 /)$
1 Sleep Medicine Center-CENC, 1070-068 Lisbon, Portugal; reis.catia@gmail.com (C.R.); amelia.feliciano71@gmail.com (A.F.); hugo.simiao@gmail.com (H.C.-S.); tania.gaspar.barra@gmail.com (T.G.); joapim@gmail.com (J.P.); joanavazdecastro@gmail.com (J.V.C.); sofia.rebocho@gmail.com (S.R.)

2 Instituto de Saúde Ambiental (ISAMB), Faculdade de Medicina, Universidade de Lisboa, 1649-026 Lisbon, Portugal; ginatome@sapo.pt (G.T.); catiasofiabranquinho@gmail.com (C.B.); lisramiro@sapo.pt (L.R.); smsgaspar@gmail.com (S.G.); margarida.gaspardematos@gmail.com (M.G.M.)

3 Comprehensive Health Research Center (CHRC), Nova Medical School, Universidade Nova de Lisboa, 1169-056 Lisbon, Portugal; raquel@ufp.edu.pt (M.R.S.); lmgp@uevora.pt (L.P.); helena.canhao@nms.unl.pt (H.C.)

4 Faculdade de Medicina de Lisboa, Instituto de Medicina Molecular João Lobo Antunes (IMM), 1649-028 Lisbon, Portugal; ccbentes@gmail.com

5 Católica Research Centre for Psychological Family and Social Wellbeing (CRC-W), Catholic University, 1649-023 Lisbon, Portugal

6 Clínica Lusíadas de Almada/Parque das Nações-Lisboa 2810-500, 1990-095 Almada, Portugal

7 Psychiatry and Mental Health Department-Centro Hospitalar de Lisboa Ocidental (CHLO), 1349-019 Lisbon, Portugal

8 Departamento de Medicina, Serviço de Pneumologia, Centro Hospitalar Póvoa de Varzim/Vila do Conde, 4490-421 Póvoa do Varzim, Portugal; maugustamachado68@gmail.com

9 Centro Lusíada de Investigação em Serviço Social e Intervenção Social (CLISSIS), Universidade Lusíada, 1349-001 Lisboa, Portugal

10 Faculty Human Kynetics, Universidade de Lisboa, 1495-751 Lisboa, Portugal

11 Faculty of Health Sciences/Higher School of Health, University Fernando Pessoa/Fundação Fernando Pessoa, 4200-150 Porto, Portugal; fsampaio@ufp.edu.pt

12 Escola Secundária Poeta Al Berto, 7520-902 Sines, Portugal

13 Escola Superior de Saúde Atlântica, Universidade Atlântica, 2730-036 Barcarena, Portugal

14 Department of Neurology, CHULN-Centro Hospitalar Universitário Lisboa Norte, 1649-028 Lisbon, Portugal

15 Neurology and Dental Medicine, Hospital da Luz, 1500-065 Lisbon, Portugal; gabrielapsv@yahoo.com

16 Innovation \& Development in Nursing research group, CINTESIS-Center for Health Technology and Services Research, 4200-450 Porto, Portugal

17 Nursing Department, Escola Superior de Enfermagem S. João de Deus (ESESJD), University Évora, 7000-811 Évora, Portugal

18 Instituto de Sono e Medicina Dentária (ISMD), 9000-098 Funchal, Portugal; conceicaofrancopereira@gmail.com

19 Unidade do Sono, Hospital do Divino Espírito Santo de Ponta Delgada, 9500-370 Ponta Delgada, Portugal; alexandracarreiro@hotmail.com

20 Pneumologia, Hospital dos Lusíadas, 1500-458 Lisbon, Portugal; susanalmoreira@gmail.com

21 Centro Medicina Sono e Serviço Neurologia e Neurofisiologia, Hospital CUF Coimbra, 3000-600 Coimbra, Portugal; isabeluzeiro@gmail.com

22 Serviço Neurologia e Neurofisiologia, Hospital CUF Coimbra, 3000-600 Coimbra, Portugal

23 Escola Superior de Tecnologia da Saúde de Coimbra, 3046-854 Coimbra, Portugal

24 Pneumologia, Hospital de Vila Franca de Xira, 2600-009 Vila Franca de Xira, Portugal

25 Orisclinic - Centro Integrado de Medicina Dentária de Coimbra, 3030 Coimbra, Portugal; jfonsecas@hotmail.com

26 Linde Saúde-Homecare, Linde Saúde, 1800-217 Lisbon, Portugal; ana.bernarda@linde.com

27 UFRN-AMBSONO Sleep Clinic, Department Psychology, Federal University Rio Grande do Norte, Natal 59030-180, Brazil; katie.almondes@gmail.com 
* Correspondence: teresapaiva0@gmail.com

\begin{abstract}
Objective: The aim of this study was to evaluate sleep and awakening quality (SQ and AQ) during COVID-19 in a large and diversified population in order to identify significant associations and risks in terms of demography, health and health-related behaviors, sleep variables, mental health, and attitudes. Methods/Results:Online surveys were used for data collection, received from 5479 individuals from the general population, sleep disorder patients, and COVID-involved (medical doctors (MDs) and nurses) and COVID-affected professionals (teachers, psychologists, and dentists). SQ and AQ were worse in adults, females, and high-education subjects. Feeling worse, having economic problems, depression, anxiety, irritability, and a high Calamity Experience Check List (CECL) score during COVID were significantly associated with poor SQ and AQ. Shorter sleep duration, increased latency, poor nutrition, low physical activity, increased mobile and social network use, more negative and less positive attitudes and behaviors were associated with poor AQ. Conclusions: The SQ logistic regression showed gender, morbidities, CECL, and awakenings as relevant, whereas, for $\mathrm{AQ}$, relevant variables further included age and physical activity. Aiming to have a high stress compliance, each individual should sleep well, have important control of their mood, practice positive behaviors while dismissing negative behaviors and attitudes, practice exercise, have adequate nutrition, and beware of technologies and dependences.
\end{abstract}

Keywords: health; COVID-19; sleep/awakening quality; Calamity Experience Check List; mood; attitudes; health-related behaviors; dependences; health professionals; sleep patients

\title{
1. Introduction
}

Sleep is critical for mental physical health and for survival. Sleep becomes particularly relevant when facing stressing situations such as those faced during the COVID-19 pandemic [1,2].

Sleep quality (SQ) is considered a basic sleep variable impacting the individual's daily wellbeing. It may be considered a subjective evaluation item of sleep satisfaction or may be objectively quantified by polysomnography and actigraphy. The subjective dimension is quantifiable by several instruments, among which the Pittsburg Sleep Quality Index (PSQI) emerges as the international standard [3]. Nevertheless, the Jenkins Sleep Scale [4], the Medical Outcomes Study-Sleep Scale (MOS-SS) [5], the Insomnia Severity Scale [6], the Visual Analogue [7], or the Likert Scale are also valid instruments that assess subjective $\mathrm{SQ}$.

The Self Rating Questionnaire for Sleep Quality evaluates SQ, AQ, and somatic complaints in 20 questions with four levels per question, which altogether provide a single score [8]. This scale has been used in several polysomnographic studies, but the correlations with objective sleep parameters are moderate, and no distinction exists between sleep and awakening quality [9].

The objective sleep continuity variables (sleep latency, number of awakenings $<5 \mathrm{~min}$, wake after sleep onset (WASO), and sleep efficiency) are good indicators of SQ 10]. The National Sleep Foundation addresses exact value ranges associated with good or poor SQ, with some of them being used for all ages, while others vary according to age [10].

During the COVID-19 pandemic, SQ was particularly affected. A study involving Greece, Switzerland, Austria, Germany, France, and Brazil reported worse SQ in 31.3\% of participants, and $15 \%$ of them characterized their sleep as bad [11]. Poor SQ prevalence varied between $18.4 \%$ in China [12] and much higher numbers in Italy, 55.3\% [13] and $57.1 \%$ [14]. However, $75.2 \%$ of Chinese adults in home isolation for more than 77 days rated their SQ as very good [15]. This might suggest that some cultural and/or regional differences might also affect subjective SQ [16,17].

The factors associated with poor sleep quality are anxiety, stress, or depression [10,11,13-24], marked changes in sleep/wake rhythm [10,18,25], insomnia [18], not exercising [19,23], 
increased use of multimedia such as television (TV) viewing and high personal computer (PC) use [23], negative attitude toward COVID-19 control measures [26], higher education level [19,26], family burden [24], low social capital [13,19], reduced flexibility [27], negative mood and "shielding" from the virus [24,28], COVID-19-related worries, or decreased resilience [29].

Protective factors are high social capital [13], social support, staying busy, and using free time in relaxing activities [30]. Furthermore, SQ mediates the relationship between physical activity and quality of life (QoL) [12].

The more affected groups were healthcare workers, mostly those working in frontline services [7,14,24,31-33], young people [11], older age [7], females [10,11,18,19], males performing highly demanding jobs [34], people suffering from death in the family [20], or those living in places with high COVID-19 prevalence [14].

Despite the predominantly negative impact upon SQ, some data suggest that the lockdown effect is bimodal since this factor improves in a subset of individuals $[35,36]$. This occurred in subsets of adults [12], particularly insomniacs. Morning awakening is a critical functional period due to the association with sleep inertia, sleep restoration, increased blood pressure and cortisol circadian rhythms, increased risk of morning headaches [37], awakening epilepsies, cardio- or cerebrovascular acute disorders, and sleep-awakening symptoms. Poor quality of awakening is often associated with excessive daytime sleepiness [38], insomnia symptoms, sleep/wake phase delay syndrome [39], sleep deprivation/insufficient sleep, and nonrestorative sleep [40]. In spite of being a key factor for individuals' daily wellbeing, the quality of morning awakening is not currently investigated in population surveys.

The aim of this study was to characterize the impact of the COVID-19 pandemic on sleep and awakening qualities of a large, diversified population. A further objective was to identify significant associations and risks in terms of demography, health, other sleep variables, confinement mood, attitudes, behaviors, mood scales (depression, anxiety, irritability, and worries), economic problems, and health-related/risk behaviors (physical activity, multimedia use, nutrition, toxic habits, and addictions, i.e., habits, practices, activities, or personal attributes that either enhance or put at risk the overall health).

\section{Materials and Methods}

The Survey Legend ${ }^{\circledR}$ platform was used. Surveys were anonymous, for adults (>18 years), allowing data analysis and statistical use. The first page included purpose, authors, ethical reference, contact person, and supporting entities. It was online during the first COVID-19 wave, from April to August 2020. Although the surveys were adapted to each main subgroup, they all had a core and common structure used in this study.

The overall project was approved by CENC's Ethical Committee 1/2020. There was no funding, public or private, and no conflict of interests.

Online surveys were used, and 5479 individuals were included and distributed as follows: the general population (GP) $(N=972 ;$ age $=49.2 \pm 13.5 ; 77.1 \%$ females $)$; sleep disorder patients (SDPs) $(N=1261 ;$ age $=57.8 \pm 14.2 ; 42.2 \%$ females $)$; professionals COVID-involved: medical doctors and nurses $(N=2794$; age $=44.8 \pm 13.4 ; 72.7 \%$ females $)$; professionals COVID-affected: teachers, psychologists, and dentists $(N=452$; age $=45.0 \pm 8.9 ; 88.1 \%$ females). Age and gender differences of the subgroups are considered an important issue, since they achieve population diversity. The study includes data obtained in the Portuguese mainland and islands (Madeira and Azores).

Surveys addressed the following topics: demographics, health status; confinement mood, attitudes, and behaviors; Calamity Experience Check List (CECL); sleep; physical activity (PA); multimedia use; nutrition; toxic habits and addictions.

Demographics, in addition to conventional information, included the number of people living together during the pandemic.

Health status included yes/no questions to the following topics: being healthy (subjective) or suffering from sleep, psychiatric, neurologic, cardiovascular, respiratory, aller- 
gies, gastrointestinal, rheumatologic, endocrinologic/metabolic, autoimmune, orthopedic, cancer, renal, dermatologic, hematologic, gynecologic, urologic, ear/nose/throat (ENT), or ophthalmologic disorders, chronic pain, fatigue, and dizziness.

The morbidities index (MI) is the sum of all referred morbidities at baseline with respect to COVID-19 in terms of worsening (morbidities worsening index (MWI)) and improvement (morbidities improvement index (MII)).

Confinement attitudes and behaviors were evaluated by yes/no answers. The average and number of both positive and negative attitudes and behaviors were computed per subject and used in this study. Positive attitudes were the following: felt OK; had less stress; made important discoveries. Negative attitudes were the following: fed up or tired; cannot stand it; loneliness; missing family or friends; felt in imprisonment/claustrophobia: had worries and fears; had unexpected conflicts; cannot stand companion; cannot stand children; cannot stand elderly; fed up with the children's tele-school. Positive behaviors were the following: tidying up; new type of work: phone friends; decided life changes; wrote a book, articles, or memories; learned new abilities; gardening/agriculture; invented funny or spiritual things; worked; walking/gym/sports; reading/music/studying; domestic work. Negative behaviors were the following: developed new addictions; get bored; mourned all time; slept as much as possible.

Calamity mood data were obtained using 1-10 VASs (visual analogue scales). The Calamity Experience Check List (CECL) was computed by averaging, for each subject, the scales of depression, anxiety, irritability, and worries (Tomé et al. in publication). The reason for such a selection is related to the fact that these symptoms, in clinical practice, are quite frequently associated with sleep complaints.

Sleep data included the following data relative to weekdays and weekends during COVID-19: sleep schedules, subjective sleep duration in hours, sleep latency in minutes, number of awakenings, and sleep quality (SQ) and awakening quality (AQ) obtained using a 1-10 VAS.

Physical activity was quantified in terms of intensity (null, mild, moderate, and intense) and frequency (hours/week).

Screen time (television, social networks, mobile phone, and gaming use) was quantified in hours/day.

Nutrition included daily meals, and scores for the recommended intake frequencies were calculated [41] (Appendix A).

Toxic habits-Smoking and alcoholic consumption were categorized as yes/no, and daily cigarettes and glasses of beer, wine, aperitive wine, and brandy were assessed, respectively. Drug use was quantified as no, occasionally, sometimes, or regularly

Statistics-Qualitative variables were described by absolute and relative frequencies, while quantitative variables were calculated as the mean or median depending on data distribution. Normality was tested by the Kolmogorov-Smirnov test. Most continuous variables had a normal distribution except for CECL, get up time for weekdays and weekends, and cigarettes per/day. The 25th percentile (P25) values for SQ and AQ were calculated for the pre-COVID-19 period, and the variables were dichotomized as P25 or below (poor quality) and $>$ P25 (good quality). The effect of the variables of interest was evaluated according to the variables in question using a paired $t$-test to compare SQ and AQ before (routinely occurring prior to the pandemic) and during COVID-19, chi-square tests (qualitative answers/frequency tables), and ANOVA (unidirectional analysis of variance) with post hoc Bonferroni tests. A logistic regression model was performed for SQ and AQ with age, sex, CECL, number of morbidities, sleep latency, sleep awakenings on weekdays during COVID-19, and physical activity intensity as covariates. All tests were performed using SPSS ${ }^{\circledR} \mathrm{v} 25$. Statistical significance was set at 0.05 . 


\section{Results}

3.1. $S Q$ and $A Q$ before and during COVID-19

Data from SQ and AQ before and during COVID-19 were compared; they are shown in Table 1.

Table 1. Data from sleep quality (SQ) and awakening quality (AQ) before and during COVID-19.

\begin{tabular}{|c|c|c|c|c|c|}
\hline Variable & Value & SQ Pre COVID & SQCOVID & AQ Pre COVID & AQ COVID \\
\hline \multirow[t]{2}{*}{ Number } & Valid & 4230 & 4232 & 4227 & 4223 \\
\hline & Missing & 1249 & 1247 & 1252 & 1256 \\
\hline Median & & 7 & 6 & 7 & 6 \\
\hline Minimum & & 1 & 1 & 1 & 1 \\
\hline Maximum & & 10 & 10 & 10 & 10 \\
\hline \multirow[t]{4}{*}{ Percentiles } & 25 & 5 & 4 & 5 & 4 \\
\hline & 50 & 7 & 6 & 7 & 6 \\
\hline & 75 & 8 & 7 & 8 & 7 \\
\hline & 90 & 9 & 8 & 9 & 8 \\
\hline
\end{tabular}

The reduction in SQ and AQ during COVID-19 was significant: $t=32,456, n=4222$, $p<0.001$ and $t=25.06, n=4213, p<0.001$, respectively. Before COVID-19, 21.9\% had poor SQ; this number increased to 36.3\% during COVID-19; the values for AQ were $22.8 \%$ before and $34.6 \%$ during the COVID-19 period. However, comparing before and during COVID-19 periods, $4.2 \%$ and $4.8 \%$ of the individuals improved their SQ and AQ, and $44.6 \%$ and $48.8 \%$ changed neither of them.

\section{2. $S Q$ and $A Q$ during COVID-19}

3.2.1. Demographic Data

Differences considering demographic data are shown in Table 2. SQ and AQ were worse for adults (30-64 years), females, and subjects with higher education. Differences concerning civil status were only significant for $A Q$, which were better for married people $\left(\chi^{2}=19.767 ; p=0.001\right)$.

\subsubsection{Mood and Problems}

The differences between the two groups (poor and good SQ and AQ) were significant for mood and economic problems.

During the 2020 confinement, lower values (feeling worse) were significant for both poor SQ and poor AQ; in terms of the economic problems, depression, anxiety, irritability, and the CECL, these were increased (i.e., more problems/more depressed, etc.) for poor SQ and AQ (Table 3).

Table 2. Associations of sleep and awakening quality with demographic data.

\begin{tabular}{|c|c|c|c|c|c|c|}
\hline \multicolumn{2}{|c|}{ Age Groups } & \multirow{2}{*}{$\begin{array}{c}\text { Young } \\
166 \\
(3.0)\end{array}$} & \multirow{2}{*}{$\begin{array}{c}\text { Adults } \\
1544 \\
(28.3)\end{array}$} & \multirow{2}{*}{$\begin{array}{c}\text { Elderly } \\
269 \\
(4.9)\end{array}$} & \multirow{2}{*}{$\begin{array}{c}\text { Total (\%) } \\
1979(36.3)\end{array}$} & \multirow{2}{*}{$\begin{array}{c}p \text {-Value } \\
<0.001\end{array}$} \\
\hline Sleep & Poor & & & & & \\
\hline Quality P25 & Good & $\begin{array}{l}317 \\
(5.8)\end{array}$ & $\begin{array}{l}2516 \\
(46.1)\end{array}$ & $\begin{array}{c}645 \\
(11.8)\end{array}$ & 3478 (63.7) & \\
\hline \multirow{2}{*}{$\begin{array}{l}\text { Awakening } \\
\text { Quality P25 }\end{array}$} & Poor & $\begin{array}{c}165 \\
(3.0)\end{array}$ & $\begin{array}{l}1499 \\
(27.5)\end{array}$ & $\begin{array}{c}224 \\
(4.1)\end{array}$ & 1888 (34.6) & \multirow[t]{2}{*}{$<0.001$} \\
\hline & Good & $\begin{array}{c}318 \\
(5.8)\end{array}$ & $\begin{array}{c}2561 \\
(47.0)\end{array}$ & $\begin{array}{c}690 \\
(12.6)\end{array}$ & 3569 (65.4) & \\
\hline \multicolumn{2}{|c|}{ Total (\%) } & $\begin{array}{c}483 \\
(8.8)\end{array}$ & $\begin{array}{c}4060 \\
(74.4)\end{array}$ & $\begin{array}{c}914 \\
(16.8)\end{array}$ & $\begin{array}{c}5457 \\
(100.0)\end{array}$ & \\
\hline
\end{tabular}


Table 2. Cont.

\begin{tabular}{|c|c|c|c|c|c|c|c|c|c|c|}
\hline \multicolumn{2}{|c|}{ Age Groups } & Young & Adults & Elderly & Total (\%) & $p$-Value & & & & \\
\hline \multicolumn{2}{|c|}{ Gender } & Male & Female & $\begin{array}{c}\text { Total } \\
(\%)\end{array}$ & $p$-Value & & & & & \\
\hline \multirow{2}{*}{$\begin{array}{c}\text { Sleep } \\
\text { Quality P25 }\end{array}$} & Poor & $\begin{array}{c}505 \\
(9.2)\end{array}$ & $\begin{array}{l}1482 \\
(27.1)\end{array}$ & $\begin{array}{l}1987 \\
(36.3)\end{array}$ & $<0.001$ & & & & & \\
\hline & Good & $\begin{array}{l}1264 \\
(23.1)\end{array}$ & $\begin{array}{l}2228 \\
(40.6)\end{array}$ & $\begin{array}{l}3492 \\
(63.7)\end{array}$ & & & & & & \\
\hline \multirow{2}{*}{$\begin{array}{l}\text { Awakening } \\
\text { Quality P25 }\end{array}$} & Poor & $\begin{array}{l}486 \\
(8.9)\end{array}$ & $\begin{array}{l}1408 \\
(25.7)\end{array}$ & $\begin{array}{l}1894 \\
(34.6)\end{array}$ & $<0.001$ & & & & & \\
\hline & Good & $1283(23.4)$ & $\begin{array}{c}2302 \\
(42.0)\end{array}$ & $\begin{array}{c}3585 \\
(65.4)\end{array}$ & & & & & & \\
\hline \multicolumn{2}{|c|}{ Total (\%) } & $\begin{array}{l}1769 \\
(32.3)\end{array}$ & $\begin{array}{l}3710 \\
(67.7)\end{array}$ & $\begin{array}{l}5479 \\
(100.0)\end{array}$ & & & & & & \\
\hline \multicolumn{2}{|c|}{ Education } & Low & High & $\begin{array}{c}\text { Total } \\
(\%)\end{array}$ & $p$-Value & & & & & \\
\hline \multirow{2}{*}{$\begin{array}{c}\text { Sleep } \\
\text { Quality P25 }\end{array}$} & Poor & $\begin{array}{c}268 \\
(4.9)\end{array}$ & $\begin{array}{c}1719 \\
(31.4)\end{array}$ & $\begin{array}{c}1987 \\
(36.3)\end{array}$ & $<0.001$ & & & & & \\
\hline & Good & $\begin{array}{c}624 \\
(11.4)\end{array}$ & $\begin{array}{c}2868 \\
(52.3)\end{array}$ & $\begin{array}{c}3492 \\
(63.7)\end{array}$ & & & & & & \\
\hline \multirow{2}{*}{$\begin{array}{l}\text { Awakening } \\
\text { Quality P25 }\end{array}$} & Poor & $\begin{array}{l}232 \\
(4.2)\end{array}$ & $\begin{array}{l}1662 \\
(30.4)\end{array}$ & \multirow{2}{*}{\multicolumn{2}{|c|}{$\begin{array}{l}1894(34.6)<0.001 \\
3585 \\
(65.4)\end{array}$}} & & & & & \\
\hline & Good & $\begin{array}{c}660 \\
(12.1)\end{array}$ & $\begin{array}{c}2925 \\
(53.3)\end{array}$ & & & & & & & \\
\hline \multicolumn{2}{|c|}{ Total (\%) } & $\begin{array}{c}892 \\
(16.3) \\
\end{array}$ & $\begin{array}{c}4587 \\
(83.7) \\
\end{array}$ & $\begin{array}{l}5479 \\
(100.0)\end{array}$ & & & & & & \\
\hline \multicolumn{2}{|c|}{ Civil status } & Married & Single & Widow & Divorced & Union & Total (\%) & $p$-Value & & \\
\hline \multirow{2}{*}{$\begin{array}{c}\text { Sleep } \\
\text { Quality P25 }\end{array}$} & Poor & $\begin{array}{l}1016 \\
(18.6)\end{array}$ & $458(8.4)$ & $\begin{array}{c}50 \\
(0.9)\end{array}$ & $206(3.8)$ & 255 (4.6) & 1987 (36.3) & \multirow[t]{2}{*}{0.316} & & \\
\hline & Good & $\begin{array}{c}1876 \\
(34.2)\end{array}$ & $\begin{array}{c}790 \\
(14.4) \\
\end{array}$ & $\begin{array}{c}85 \\
(1.6) \\
\end{array}$ & $315(5.7)$ & $422(7.8)$ & 3492 (63.7) & & & \\
\hline \multirow{2}{*}{$\begin{array}{l}\text { Awakening } \\
\text { Quality P25 }\end{array}$} & Poor & $\begin{array}{c}928 \\
(17.0)\end{array}$ & 472 (8.6) & $\begin{array}{c}41 \\
(0.7)\end{array}$ & 196 (3.6) & $255(4.7)$ & 1894 (34.6) & \multirow[t]{2}{*}{$<0.001$} & & \\
\hline & Good & $\begin{array}{c}1964 \\
(35.8)\end{array}$ & $\begin{array}{c}776 \\
(14.2)\end{array}$ & $\begin{array}{c}94 \\
(1.7)\end{array}$ & $325(5.9)$ & 422 (7.7) & 3585 (65.4) & & & \\
\hline \multicolumn{2}{|c|}{ Total (\%) } & $\begin{array}{c}2892 \\
(52.8)\end{array}$ & $\begin{array}{l}1248 \\
(22.8)\end{array}$ & $\begin{array}{l}135 \\
(2.5)\end{array}$ & $521(9.5)$ & $\begin{array}{c}677 \\
(12.4)\end{array}$ & $\begin{array}{c}5479 \\
(100.0) \\
\end{array}$ & & & \\
\hline \multicolumn{2}{|c|}{$\begin{array}{c}\text { People at } \\
\text { theHousehold }\end{array}$} & \multicolumn{2}{|c|}{1} & 2 & 3 & 4 & 5 & $\geq 6$ & Total (\%) & $p$-Value \\
\hline \multirow{2}{*}{$\begin{array}{c}\text { Sleep } \\
\text { Quality P25 }\end{array}$} & Poor & \multicolumn{2}{|c|}{$324(6.3)$} & $\begin{array}{c}645 \\
(12.5)\end{array}$ & $436(8.5)$ & 385 (7.5) & $108(2.1)$ & $76(1.5)$ & $1974(38.3)$ & \multirow[t]{2}{*}{0.029} \\
\hline & Good & 439 & (8.5) & $\begin{array}{c}1173 \\
(22.8)\end{array}$ & 685 (13.3) & $\begin{array}{c}605 \\
(11.7)\end{array}$ & $167(3.2)$ & $113(2.2)$ & 3182 (61.7) & \\
\hline \multirow{2}{*}{$\begin{array}{l}\text { Awakening } \\
\text { Quality P25 }\end{array}$} & Poor & 322( & $(6.2)$ & $\begin{array}{c}631 \\
(12.2)\end{array}$ & 408 (6.9) & $358(6.9)$ & $255(4.7)$ & $65(1.3)$ & $1886(36.6)$ & \multirow[t]{2}{*}{0.018} \\
\hline & Good & $441(1$ & 13.5) & $\begin{array}{c}1187 \\
(23.0)\end{array}$ & $632(12.3)$ & $\begin{array}{c}632 \\
(12.3)\end{array}$ & 422 (7.7) & $124(2.4)$ & $3270(63.4)$ & \\
\hline \multicolumn{2}{|c|}{ Total (\%) } & \multicolumn{2}{|c|}{$763(14.8)$} & $\begin{array}{c}1818 \\
(35.3)\end{array}$ & 1121 (21.7) & $\begin{array}{c}990 \\
(19.2)\end{array}$ & 275 (12.4) & $\begin{array}{c}189 \\
(3.7 .0)\end{array}$ & \multicolumn{2}{|c|}{$5156(100 \%)$} \\
\hline
\end{tabular}

Pearson's chi-square test for all group comparisons; values are given as the frequency (percentage); P25-represents the 25th percentile of the score distribution; young- $\leq 29$ years; adults-30-64 years; elderly- $\geq 65$ years. 
Table 3. Differences in sleep and awakening quality relative to feelings and mood during COVID-19.

\begin{tabular}{|c|c|c|c|c|c|c|c|c|}
\hline Sleep Quality P25 & & $N$ & Mean & SE & Minimum & Maximum & $Z$ & $p$-Value \\
\hline \multirow{2}{*}{ How are you living during confinement } & Poor & 1168 & 6.31 & 6.21 & 1 & 10 & 33.025 & $<0.001$ \\
\hline & Good & 3774 & 6.66 & 6.60 & 1 & 10 & & \\
\hline \multirow{2}{*}{ How are your economic problems? } & Poor & 1186 & 3.38 & 3.25 & 1 & 10 & 51.831 & $<0.001$ \\
\hline & Good & 3790 & 2.87 & 2.80 & 1 & 10 & & \\
\hline \multirow{2}{*}{ How is your depression? } & Poor & 1190 & 4.17 & 4.03 & 1 & 10 & 42.987 & $<0.001$ \\
\hline & Good & 3819 & 3.65 & 3.58 & 1 & 10 & & \\
\hline \multirow{2}{*}{ How is your anxiety? } & Poor & 1190 & 5.13 & 4.99 & 1 & 10 & 42.71 & $<0.001$ \\
\hline & Good & 3804 & 4.58 & 4.50 & 1 & 10 & & \\
\hline \multirow{2}{*}{ How is your irritability? } & Poor & 1186 & 4.95 & 4.81 & 1 & 10 & 18.996 & $<0.001$ \\
\hline & Good & 3817 & 4.58 & 4.50 & 1 & 10 & & \\
\hline \multirow{2}{*}{ How are your worries vs. uncertainty? } & Poor & 1185 & 6.37 & 6.23 & 1 & 10 & 25.407 & $<0.001$ \\
\hline & Good & 3788 & 5.96 & 5.88 & 1 & 10 & & \\
\hline \multirow{2}{*}{ Calamity Experience Check List } & Poor & 1197 & 5.11 & 5.00 & 1 & 10 & 47.941 & $<0.001$ \\
\hline & Good & 3847 & 4.64 & 4.58 & 0.75 & 10 & & \\
\hline Awakening Quality P25 & & $N$ & Mean & SE & Minimum & Maximum & $Z$ & $p$-Value \\
\hline \multirow{2}{*}{ How are you living during confinement? } & Poor & 1845 & 6.03 & 1.784 & 1 & 10 & 290.166 & $<0.001$ \\
\hline & Good & 3097 & 6.91 & 1.741 & 1 & 10 & & \\
\hline \multirow{2}{*}{ How are your economic problems? } & Poor & 1878 & 3.33 & 2.237 & 1 & 10 & 74.496 & $<0.001$ \\
\hline & Good & 3098 & 2.79 & 2.06 & 1 & 10 & & \\
\hline \multirow{2}{*}{ How is your depression? } & Poor & 1879 & 4.58 & 2.353 & 1 & 10 & 364.663 & $<0.001$ \\
\hline & Good & 3130 & 3.3 & 2.266 & 1 & 10 & & \\
\hline \multirow{2}{*}{ How is your anxiety? } & Poor & 1883 & 5.6 & 2.39 & 1 & 10 & 408.062 & $<0.001$ \\
\hline & Good & 3111 & 4.17 & 2.439 & 1 & 10 & & \\
\hline \multirow{2}{*}{ How is your irritability? } & Poor & 1879 & 5.51 & 2.42 & 1 & 10 & 359.52 & $<0.001$ \\
\hline & Good & 3124 & 4.16 & 2.443 & 1 & 10 & & \\
\hline \multirow{2}{*}{ How are your worries vs. uncertainty? } & Poor & 1878 & 6.74 & 2.236 & 1 & 10 & 254.552 & $<0.001$ \\
\hline & Good & 3095 & 5.64 & 2.457 & 1 & 10 & & \\
\hline \multirow{2}{*}{ Calamity Experience Check List } & Poor & 1892 & 5.57 & 1.92 & 1 & 10 & 530.367 & $<0.001$ \\
\hline & Good & 3152 & 4.26 & 1.98 & 0.75 & 10 & & \\
\hline
\end{tabular}

P25-represents the 25th percentile of the score distribution; SE-standard error; Z-scores indicate the differences between the groups.

\subsubsection{Sleep}

Fewer differences were observed in sleep variables for the comparisons between poor and good SQ and AQ (Table 4).

For SQ, only the get up time on weekdays (later for poor SQ) and the higher values of sleep latency and awakenings, both on weekdays and weekends, were significantly different.

For $A Q$, differences concerned mostly weekdays in relation to earlier get up time, shorter sleep duration, and longer sleep latency.

\subsubsection{Health-Related and Risks Behaviors}

Health-related behaviors (HRBs) are presented in Table 5. For SQ data, these results were ambiguous since participants with poor SQ ate more recommended and less nonrecommended food, smoked less, and had lower levels of TV and game dependence.

However, for $A Q$, the data are quite clear, i.e., participants with poor $A Q$ practiced less PA, while they ate fewer meals and less recommended food and more non-recommended food, with a poor yes/no proportion; they had higher use of social networks and mobile phones, as well as a higher dependence of social networks, but they consumed fewer alcoholic drinks.

\subsubsection{Attitudes and Behaviors}

The associations between attitudes and SQ/AQ are detailed in Table 6 . 
Poor SQ was associated with negative attitudes and behaviors, while AQ was significantly lower in terms of negative attitudes and higher in terms of negative behaviors and positive attitudes and behaviors, as well as in negative behaviors, which was unexpected.

Table 4. Differences in sleep variables for the comparisons between poor and good SQ and AQ during COVID.

\begin{tabular}{|c|c|c|c|c|c|c|c|c|}
\hline Sleep Quality P25 & & $N$ & Mean & SE & Minimum & Maximum & $Z$ & $p$-Value \\
\hline \multirow{2}{*}{ Get up time weekdays (hours) } & Poor & 1007 & 8.05 & 1.535 & 3.75 & 19.25 & 15.825 & $<0.001$ \\
\hline & Good & 3449 & 7.85 & 1.428 & 3.00 & 19.00 & & \\
\hline \multirow{2}{*}{ Get up time weekends (hours) } & Poor & 1012 & 9.00 & 1.511 & 5.00 & 15.00 & 3.732 & 0.053 \\
\hline & Good & 3461 & 8.90 & 1.506 & 3.25 & 19.00 & & \\
\hline \multirow{2}{*}{ Time into bed weekdays (hours) } & Poor & 980 & 0.00 & 1.548 & 18.00 & 8.00 & 2.863 & 0.091 \\
\hline & Good & 3342 & 23.51 & 1.516 & 18.00 & 15.00 & & \\
\hline \multirow{2}{*}{ Time into bed weekends (hours) } & Poor & 986 & 0.35 & 1.659 & 18.00 & 11.50 & 3.716 & 0.054 \\
\hline & Good & 3330 & 0.24 & 1.603 & 18.00 & 12.00 & & \\
\hline \multirow{2}{*}{ Sleep duration weekdays (hours) } & Poor & 980 & 6.69 & 1.615 & 00.25 & 16.00 & 0.042 & 0.838 \\
\hline & Good & 3302 & 6.68 & 1.641 & 00.13 & 16.00 & & \\
\hline \multirow{2}{*}{ Sleep duration weekends (hours) } & Poor & 975 & 7.41 & 1.849 & 00.34 & 16.00 & 0.881 & 0.348 \\
\hline & Good & 3295 & 7.48 & 1.973 & 00.13 & 19.00 & & \\
\hline \multirow{2}{*}{ Sleep latency weekdays (min) } & Poor & 928 & 34.16 & 35.017 & 0 & 300 & 3.937 & 0.047 \\
\hline & Good & 3153 & 31.68 & 32.972 & 0 & 240 & & \\
\hline \multirow{2}{*}{ Sleep latency weekends (min) } & Poor & 927 & 34.06 & 35.841 & 0 & 300 & 5.6 & 0.018 \\
\hline & Good & 3140 & 31.04 & 33.693 & 0 & 302 & & \\
\hline \multirow{2}{*}{ Night awakenings weekdays (number) } & Poor & 802 & 3.00 & 3.128 & 1 & 30 & 7.158 & 0.007 \\
\hline & Good & 2524 & 2.72 & 2.451 & 0.5 & 30 & & \\
\hline \multirow{2}{*}{ Night awakenings weekends (number) } & Poor & 760 & 2.59 & 2.260 & 1 & 30 & 9.994 & 0.002 \\
\hline & Good & 2420 & 2.34 & 1.689 & 0.1 & 30 & & \\
\hline Awakening Quality P25 & & $N$ & Mean & SE & Minimum & Maximum & $Z$ & $p$-Value \\
\hline \multirow{2}{*}{ Get up time weekdays (hours) } & Poor & 1589 & 7.83 & 1.47 & 3.00 & 19.25 & 4.799 & 0.029 \\
\hline & Good & 2867 & 7.93 & 1.45 & 3.00 & 17.00 & & \\
\hline \multirow{2}{*}{ Get up time weekends (hours) } & Poor & 1592 & 8.93 & 1.51 & 4.00 & 19.00 & 0.069 & 0.794 \\
\hline & Good & 2881 & 8.92 & 1.51 & 3.25 & 18.00 & & \\
\hline \multirow{2}{*}{ Time into bed weekdays (hours) } & Poor & 1545 & 23.51 & 1.51 & 18.00 & 09.50 & 0.426 & 0.514 \\
\hline & Good & 2777 & 23.54 & 1.53 & 18.00 & 15.00 & & \\
\hline \multirow{2}{*}{ Time into bed weekends (hours) } & Poor & 1551 & 0.28 & 1.65 & 18.00 & 12.00 & 0.141 & 0.708 \\
\hline & Good & 2765 & 0.26 & 1.60 & 18.00 & 12.00 & & \\
\hline \multirow{2}{*}{ Sleep duration weekdays (hours) } & Poor & 1540 & 6.57 & 1.58 & 0.29 & 16.00 & 12.005 & 0.001 \\
\hline & Good & 2742 & 6.75 & 1.66 & 0.13 & 16.00 & & \\
\hline \multirow{2}{*}{ Sleep duration weekends (hours) } & Poor & 1539 & 7.40 & 1.87 & 0.29 & 16.00 & 2.85 & 0.091 \\
\hline & Good & 2731 & 7.50 & 1.99 & 0.13 & 19.00 & & \\
\hline \multirow{2}{*}{ Sleep latency weekdays (min) } & Poor & 1478 & 33.82 & 34.90 & 0.00 & 300.00 & 5.165 & 0.023 \\
\hline & Good & 2603 & 31.35 & 32.59 & 0.00 & 240.00 & & \\
\hline \multirow{2}{*}{ Sleep latency weekends (min) } & Poor & 1475 & 33.09 & 34.97 & 0.00 & 300.00 & 3.68 & 0.055 \\
\hline & Good & 2592 & 30.95 & 33.76 & 0.00 & 302.00 & & \\
\hline \multirow{2}{*}{ Night awakenings weekdays (number) } & Poor & 1228 & 2.85 & 2.70 & 1.00 & 30.00 & 0.989 & 0.32 \\
\hline & Good & 2098 & 2.75 & 2.59 & 0.50 & 30.00 & & \\
\hline \multirow{2}{*}{ Night awakenings weekends (number) } & Poor & 1163 & 2.43 & 1.81 & 0.10 & 30.00 & 0.414 & 0.52 \\
\hline & Good & 2017 & 2.39 & 1.86 & 0.50 & 30.00 & & \\
\hline
\end{tabular}

P25-represents the 25th percentile of the sample distribution; SE-standard error; hours are given in decimal values for local time and in $24 \mathrm{~h}$ format; $\mathrm{Z}$-scores indicate the differences between the groups.

\subsubsection{Health Prior to and during COVID-19}

A small number of subjects $(n=122)$ were COVID-19-infected, of which 30 were asymptomatic, with poor SQ and AQ in eight and seven respondents, respectively; 81 had mild symptoms, with poor SQ and AQ in 46 and 37 respondents, respectively; seven had pneumonia, with poor SQ and AQ in four and four respondents, respectively; four were hospitalized, none of which had poor SQ or AQ. For SQ, the chi-square value was 17.561 $(p<0.002)$ and, for $\mathrm{AQ}$, the chi-square value was $8.921(p=0.063)$. 
The statistical links between SQ/AQ and body mass index (BMI)/health are presented in Table 7.

Table 5. Differences in sleep quality and awakening quality relative to health-related and risk behaviors.

\begin{tabular}{|c|c|c|c|c|c|c|c|c|}
\hline SLEEP Quality P25 & & $\mathbf{N}$ & Mean & SE & Minimum & Maximum & $\mathbf{Z}$ & $p$-Value \\
\hline \multirow{2}{*}{ Physical activity during COVID (hours) } & Poor & 905 & 2.66 & 4.027 & 0 & 48 & 0.518 & 0.472 \\
\hline & Good & 2229 & 2.77 & 3.828 & 0 & 60 & & \\
\hline \multirow{2}{*}{ Meals/day during COVID } & Poor & 1162 & 3.81 & 0.901 & 1 & 5 & 1.556 & 0.212 \\
\hline & Good & 2942 & 3.85 & 0.906 & 1 & 5 & & \\
\hline \multirow[t]{2}{*}{ Food Recommended } & Poor & 1161 & 1.3167 & 0.14009 & 1 & 2 & 2.144 & 0.143 \\
\hline & Good & 2940 & 1.31 & 0.13375 & 1 & 2 & & \\
\hline \multirow[t]{2}{*}{ Food Recommended YES } & Poor & 1147 & 5.37 & 2.324 & 0 & 14 & 4.551 & 0.033 \\
\hline & Good & 2917 & 5.26 & 2.211 & 1 & 14 & & \\
\hline \multirow{2}{*}{ Food Recommended NO } & Poor & 1154 & 11.45 & 2.452 & 1 & 17 & 4.133 & 0.042 \\
\hline & Good & 2932 & 11.63 & 2.324 & 1 & 17 & & \\
\hline \multirow{2}{*}{ Food Recommended YES/NO proportion } & Poor & 1146 & 0.5421 & 0.39774 & 0 & 4.67 & 2.144 & 0.143 \\
\hline & Good & 2915 & 0.5158 & 0.36136 & 0.06 & 4.67 & & \\
\hline \multirow[t]{2}{*}{ Alcohol during COVID } & Poor & 755 & 9.1836 & 26.8108 & 0 & 563.5 & 0.052 & 0.819 \\
\hline & Good & 1866 & 9.3843 & 17.0966 & 0 & 139.5 & & \\
\hline \multirow{2}{*}{ Number cigarettes/month during COVID } & Poor & 172 & 11.23 & 8.907 & 0 & 60 & 4.597 & 0.032 \\
\hline & Good & 376 & 12.94 & 8.563 & 0 & 40 & & \\
\hline \multirow{2}{*}{ TV/Day during COVID (hours) } & Poor & 1055 & 3.199 & 2.4342 & 0.1 & 20 & 3.044 & 0.081 \\
\hline & Good & 2687 & 3.044 & 2.4365 & 0.1 & 20 & & \\
\hline Social Networks during COVID (hours) & Poor & 927 & 2.541 & 2.4236 & 0.1 & 20 & 2.539 & 0.111 \\
\hline \multirow{2}{*}{ Mobile use/Day during COVID (hours) } & $\begin{array}{l}\text { Good } \\
\text { Poor }\end{array}$ & $\begin{array}{l}2347 \\
1008\end{array}$ & $\begin{array}{l}2.398 \\
2.649\end{array}$ & $\begin{array}{l}2.2852 \\
2.8126\end{array}$ & $\begin{array}{l}0 \\
0\end{array}$ & $\begin{array}{l}20 \\
20\end{array}$ & 1485 & 0223 \\
\hline & Good & 2624 & 2.532 & 2.5031 & 0.1 & 20 & 1.400 & 0.223 \\
\hline \multirow{2}{*}{ Games/Day during COVID (hours) } & Poor & 227 & 1.825 & 1.4432 & 0.1 & 14 & 0.526 & 0.469 \\
\hline & Good & 672 & 1.925 & 1.9114 & 0.1 & 20 & & \\
\hline \multirow{2}{*}{ TV dependence } & Poor & 1097 & 3.19 & 1.962 & 1 & 10 & 6.236 & 0.013 \\
\hline & Good & 2812 & 3.38 & 2.122 & 1 & 10 & & \\
\hline Social Networks dependence & Poor & 1098 & 3.55 & 2.312 & 1 & 10 & 2.659 & 0.103 \\
\hline & Good & 2817 & 3.68 & 2.4 & 1 & 10 & & \\
\hline Games dependence & Good & 2813 & $\begin{array}{l}1.59 \\
1.7\end{array}$ & $\begin{array}{l}1.392 \\
1.579\end{array}$ & 1 & 10 & 4.063 & 0.044 \\
\hline Alcoho & Poor & 1094 & 1.45 & 1.171 & 1 & 10 & 0.308 & 0.579 \\
\hline Alcohol & Good & 2811 & 1.47 & 1.159 & 1 & 10 & & \\
\hline AWAKENING Quality P25 & & $\mathbf{N}$ & Mean & SE & Minimum & Maximum & $\mathbf{Z}$ & $p$-Value \\
\hline Physical astivi & Poor & 1302 & 2.46 & 3.848 & 0 & 48 & 11.8 & $<0.001$ \\
\hline I nysical activ & Good & 1832 & 2.94 & 3.903 & 0 & 60 & & \\
\hline Meals/day during COVID & Poor & 1835 & 3.8 & 0.937 & 1 & 5 & 4.635 & 0.031 \\
\hline & Good & 2269 & 3.87 & 0.877 & 1 & 5 & & \\
\hline Food Recommended & Poor & 1831 & 1.2997 & 0.13788 & 1 & 2 & 26.741 & $<0.001$ \\
\hline & Good & 2270 & 1.3217 & 0.13294 & 1 & 2 & & \\
\hline Food Recommended (YES) & Poor & 1810 & 5.09 & 2.266 & 0 & 14 & 26.402 & $<0.001$ \\
\hline & Good & 2254 & 5.45 & 2.213 & 1 & 14 & & \\
\hline Food Recommended (NO) & Poor & 1824 & 11.74 & 2.437 & 1 & 17 & 14.696 & $<0.001$ \\
\hline & Good & 2262 & 11.45 & 2.292 & 1 & 17 & & \\
\hline Food Recommended YES/NO proportion & Poor & 1808 & 0.4987 & 0.37094 & 0 & 4.67 & 14.171 & $<0.001$ \\
\hline & Good & 2253 & 0.5429 & 0.372 & 0.06 & 4.67 & & \\
\hline Alcohol consumption during COVID & Poor & 1159 & 7.8347 & 22.94508 & 0 & 563.5 & 11.188 & $<0.001$ \\
\hline & Good & 1462 & 10.5091 & 17.99032 & 0 & 139.5 & & \\
\hline $\mathrm{Nu}$ & Poor & 277 & 12.92 & 8.736 & 0 & 40 & 1.983 & 0.16 \\
\hline INu & Good & 271 & 11.88 & 8.648 & 0 & 60 & & \\
\hline TV/Day during COVID (hours) & Poor & 1645 & 3.009 & 2.4141 & 0.1 & 20 & 3.094 & 0.079 \\
\hline & Good & 2097 & 3.15 & 2.4527 & 0.1 & 20 & & \\
\hline OVID (hours) & Poor & 1504 & 2.565 & 2.4988 & 0 & 20 & 8.266 & 0.004 \\
\hline 11 & Good & 1770 & 2.331 & 2.1628 & 0.1 & 20 & & \\
\hline Mobile use/Day during COVID (hours) & Poor & 1624 & 2.761 & 2.7572 & 0 & 20 & 17.003 & $<0.001$ \\
\hline & Good & 2008 & 2.405 & 2.441 & 0.1 & 20 & & \\
\hline Games/Day during COVID (hours) & Poor & 389 & 1.919 & 1.9467 & 0.1 & 20 & 0.08 & 0.777 \\
\hline & Good & 510 & 1.885 & 1.6897 & 0.1 & 20 & & \\
\hline TV dependence & Poor & 1731 & 3.3 & 2.058 & 1 & 10 & 0,689 & 0.407 \\
\hline iv aepenaence & Good & 2178 & 3.35 & 2.097 & 1 & 10 & & \\
\hline dependence & Poor & 1736 & 3.84 & 2.407 & 1 & 10 & 21.279 & $<0.001$ \\
\hline aepenaence & Good & 2179 & 3.49 & 2.34 & 1 & 10 & & \\
\hline Games dependence & Poor & 1731 & 1.69 & 1.606 & 1 & 10 & 0.916 & 0.339 \\
\hline Games dependence & Good & 2172 & 1.65 & 1.466 & 1 & 10 & & \\
\hline Alcohol dependence & Poor & 1733 & 1.48 & 1.206 & 1 & 10 & 0.509 & 0.476 \\
\hline Aiconor aepenaence & Good & 2172 & 1.46 & 1.126 & 1 & 10 & & \\
\hline
\end{tabular}

P25-represents the 25th percentile of the sample distribution; SE-standard error; hours are given in decimal values for local time and in $24 \mathrm{~h}$ format; Z-scores indicate the differences between the groups. 
Table 6. Associations between attitudes/behaviors and sleep quality/awakening quality.

\begin{tabular}{|c|c|c|c|c|c|c|c|c|}
\hline Sleep Quality P25 & & $N$ & Mean & SE & Minimum & Maximum & Z & $p$-Value \\
\hline \multirow{2}{*}{ Positive attitudes (number) } & Poor & 1198 & 0.59 & 0.766 & 0 & 3 & 2.714 & 0.1 \\
\hline & Good & 4044 & 0.55 & 0.724 & 0 & 3 & & \\
\hline \multirow{2}{*}{ Negative attitudes (number) } & Poor & 1198 & 1.21 & 1.093 & 0 & 7 & 6.164 & 0.013 \\
\hline & Good & 4044 & 1.13 & 1.045 & 0 & 7 & & \\
\hline \multirow{2}{*}{ Positive behaviors (number) } & Poor & 1198 & 1.97 & 1.519 & 0 & 7 & 0.63 & 0.427 \\
\hline & Good & 4045 & 1.93 & 1.517 & 0 & 8 & & \\
\hline \multirow{2}{*}{ Negative behaviors(number) } & Poor & 1198 & 0.65 & 0.849 & 0 & 4 & 5.611 & 0.018 \\
\hline & Good & 4045 & 0.59 & 0.811 & 0 & 5 & & \\
\hline Awakening Quality P25 & & $N$ & Mean & SE & Minimum & Maximum & $Z$ & $p$-Value \\
\hline \multirow{2}{*}{ Positive attitudes (number) } & Poor & 1890 & 0.42 & 0.655 & 0 & 3 & 102.011 & $<0.001$ \\
\hline & Good & 3352 & 0.63 & 0.765 & 0 & 3 & & \\
\hline \multirow{2}{*}{ Negative attitudes (number) } & Poor & 1890 & 1.45 & 1.128 & 0 & 7 & 263.127 & $<0.001$ \\
\hline & Good & 3352 & 0.97 & 0.972 & 0 & 7 & & \\
\hline \multirow{2}{*}{ Positive behaviors (number) } & Poor & 1890 & 1.83 & 1.491 & 0 & 7 & 15.236 & $<0.001$ \\
\hline & Good & 3353 & 2 & 1.528 & 0 & 8 & & \\
\hline \multirow{2}{*}{ Negative behaviors(number) } & Poor & 1890 & 0.70 & 0.88 & 0 & 4 & 40.237 & $<0.001$ \\
\hline & Good & 3353 & 0.55 & 0.779 & 0 & 5 & & \\
\hline
\end{tabular}

P25-represents the 25th percentile of the sample distribution; SE—standard error; Z-scores indicate the differences between the groups.

Table 7. Relationship between sleep/awakening quality and body mass index (BMI)/health during COVID-19.

\begin{tabular}{|c|c|c|c|c|c|c|c|c|}
\hline Sleep Quality P25 & & $N$ & Mean & SE & Minimum & Maximum & $Z$ & $p$-Value \\
\hline \multirow{2}{*}{ BMI } & Poor & 1191 & 25.73 & 5.01 & 13.96 & 48.48 & 0.016 & 0.898 \\
\hline & Good & 4259 & 25.76 & 5.03 & 15.32 & 68.4 & & \\
\hline \multirow{2}{*}{ Number of morbidities } & Poor & 1198 & 2.44 & 2.22 & 0 & 16 & 309.428 & $<0.001$ \\
\hline & Good & 4201 & 1.43 & 1.598 & 0 & 13 & & \\
\hline \multirow{2}{*}{ Morbidities (worse) } & Poor & 1127 & 2.1 & 2.077 & 0 & 12 & 62.212 & $<0.001$ \\
\hline & Good & 3287 & 1.6 & 1.729 & 0 & 13 & & \\
\hline \multirow{2}{*}{ Morbidities (better) } & Poor & 1122 & 0.55 & 1.276 & 0 & 11 & 23.857 & $<0.001$ \\
\hline & Good & 3276 & 0.37 & 0.974 & 0 & 9 & & \\
\hline Awakening Quality P25 & & $N$ & Mean & SE & Minimum & Maximum & $Z$ & $p$-Value \\
\hline \multirow{2}{*}{ BMI } & Poor & 1887 & 25.38 & 4.94 & 14.82 & 48.48 & 15.952 & $<0.001$ \\
\hline & Good & 3563 & 25.95 & 5.05 & 13.96 & 68.4 & & \\
\hline \multirow{2}{*}{ Number of morbidities } & Poor & 1894 & 2.09 & 2.077 & 0 & 16 & 176.466 & $<0.001$ \\
\hline & Good & 3505 & 1.42 & 1.59 & 0 & 13 & & \\
\hline \multirow{2}{*}{ Morbidities (worse) } & Poor & 1779 & 2.37 & 2.042 & 0 & 13 & 395.207 & $<0.001$ \\
\hline & Good & 2635 & 1.3 & 1.541 & 0 & 10 & & \\
\hline \multirow{2}{*}{ Morbidities (better) } & Poor & 1776 & 0.36 & 0.927 & 0 & 9 & 10.088 & 0.002 \\
\hline & Good & 2622 & 0.46 & 1.143 & 0 & 11 & & \\
\hline
\end{tabular}

P25-represents the first quartile of the sample distribution; BMI-body mass index; the number of morbidities is relative to the pre-COVID situation; morbidities worse and better are for the post-COVID situation; SE-standard error; Z-scores indicate the differences between the groups.

SQ was poorer with an increased number of morbidities, a higher level of worsening morbidities, and a higher level of improving morbidities, whereas poor AQ was associated with a lower BMI, a higher level of worsening morbidities, and a lower level of improving morbidities.

\subsection{Logistic Regression}

A logistic regression model for $\mathrm{SQ}$ and $\mathrm{AQ}$ was performed for variables significantly associated with poor SQ and poor AQ. 


\subsubsection{Poor Sleep Quality}

Male gender (odds ratio $(\mathrm{OR})=0.725 ; 95 \%$ confidence interval $(\mathrm{CI})$ : 0.596-0.882; $p=0.001)$ was the only significant protective factor for poor SQ. A higher number of morbidities (OR $=1.184 ; 95 \%$ CI: 1.121-1.252; $p<0.001)$ and a higher average CECL $(\mathrm{OR}=1.441 ; 95 \%$ CI: $1.373-1.512 ; p<0.001)$ were risk factors. Regarding sleep variables, a high number of awakenings on weekdays during COVID-19 was the only variable representing a significantly increased risk factor for poor SQ (Table 8).

Table 8. Logistic regression analysis for poor sleep quality.

\begin{tabular}{|c|c|c|c|c|c|c|c|c|}
\hline \multirow{2}{*}{ Variables } & \multirow{2}{*}{$\beta$} & \multirow{2}{*}{ SE $\beta$} & \multirow{2}{*}{ Wald's $\chi^{2}$} & \multirow{2}{*}{ df } & \multirow{2}{*}{$p$-Value } & \multirow{2}{*}{ Odds Ratio } & \multicolumn{2}{|c|}{$95 \%$ CI } \\
\hline & & & & & & & Lower & Upper \\
\hline Age & 0.001 & 0.003 & 0.145 & 1 & 0.704 & 1.001 & 0.995 & 1.008 \\
\hline Sex (male) & -0.322 & 0.1 & 10.287 & 1 & 0.001 & 0.725 & 0.596 & 0.882 \\
\hline Number morbidities & 0.169 & 0.028 & 36.185 & 1 & $<0.001$ & 1.184 & 1.121 & 1.252 \\
\hline Calamity Experience Check List & 0.365 & 0.025 & 218.207 & 1 & $<0.001$ & 1.441 & 1.373 & 1.512 \\
\hline $\begin{array}{l}\text { Sleep latency on weekdays during } \\
\text { COVID-19 }\end{array}$ & 0.001 & 0.001 & 0.276 & 1 & 0.599 & 1.001 & 0.998 & 1.003 \\
\hline $\begin{array}{c}\text { Awakenings on weekdays during } \\
\text { COVID-19 }\end{array}$ & 0.038 & 0.019 & 3.903 & 1 & 0.048 & 1.039 & 1 & 1.078 \\
\hline Physical activity intensity & & & 2.295 & 3 & 0.514 & & & \\
\hline None & -0.267 & 0.229 & 1.37 & 1 & 0.242 & 0.765 & 0.489 & 1.198 \\
\hline Light & -0.321 & 0.222 & 2.096 & 1 & 0.148 & 0.725 & 0.469 & 1.12 \\
\hline Moderate & -0.314 & 0.219 & 2.061 & 1 & 0.151 & 0.73 & 0.475 & 1.122 \\
\hline Constant & -1.866 & 0.29 & 41.513 & 1 & $<0.001$ & 0.155 & & \\
\hline
\end{tabular}

Hosmer-Lemeshow test $\chi 2(8)=6.535, p=0.588$. Abbreviations: $\mathrm{CI}$, confidence interval; df, degrees of freedom.

\subsubsection{Poor Awakening Quality}

Being older (OR $=0.993 ; 95 \%$ CI: 0.986-0.999; $p=0.033)$, male gender $(\mathrm{OR}=0.762$; 95\% CI: 0.626-0.926; $p=0.006)$, and performing light (OR $=0.624 ; 95 \%$ CI: $0.406-0.961$; $p=0.032)$ or moderate (OR $=0.612 ; 95 \%$ CI: $0.402-0.943 ; p=0.026)$ physical activities were significant protective factors for poor $\mathrm{AQ}$. A higher number of morbidities $(\mathrm{OR}=1.168$; 95\% CI: 1.107-1.233; $p<0.001)$ and a higher average calamity scale score $(\mathrm{OR}=1.398$; 95\% CI: 1.333-1.467; $p<0.001$ ) were also risk factors for poor AQ (Table 9).

Table 9. Logistic regression analysis for poor awakening quality.

\begin{tabular}{|c|c|c|c|c|c|c|c|c|}
\hline \multirow{2}{*}{ Variables } & \multirow{2}{*}{$\beta$} & \multirow{2}{*}{ SE $\beta$} & \multirow{2}{*}{ Wald's $\chi^{2}$} & \multirow{2}{*}{ df } & \multirow{2}{*}{$p$-Value } & \multirow{2}{*}{ Odds Ratio } & \multicolumn{2}{|c|}{$95 \% \mathrm{CI}$} \\
\hline & & & & & & & Lower & Upper \\
\hline Age & -0.007 & 0.003 & 4.527 & 1 & 0.033 & 0.993 & 0.986 & 0.999 \\
\hline Sex (male) & -0.272 & 0.1 & 7.431 & 1 & 0.006 & 0.762 & 0.626 & 0.926 \\
\hline Number morbidities & 0.156 & 0.027 & 32.329 & 1 & $<0.001$ & 1.168 & 1.107 & 1.233 \\
\hline Calamity Experience Check List & 0.335 & 0.024 & 190.787 & 1 & $<0.001$ & 1.398 & 1.333 & 1.467 \\
\hline $\begin{array}{l}\text { Sleep latency on weekdays during } \\
\text { COVID-19 }\end{array}$ & 0.001 & 0.001 & 0.342 & 1 & 0.559 & 1.001 & 0.998 & 1.003 \\
\hline $\begin{array}{l}\text { Awakenings on weekdays during } \\
\text { COVID-19 }\end{array}$ & 0.019 & 0.019 & 1.034 & 1 & 0.309 & 1.019 & 0.983 & 1.057 \\
\hline Physical activity intensity & & & 5.958 & 3 & 0.114 & & & \\
\hline None & -0.353 & 0.227 & 2.425 & 1 & 0.119 & 0.703 & 0.451 & 1.096 \\
\hline Light & -0.471 & 0.22 & 4.573 & 1 & 0.032 & 0.624 & 0.406 & 0.961 \\
\hline Moderate & -0.485 & 0.217 & 4.981 & 1 & 0.026 & 0.616 & 0.402 & 0.943 \\
\hline Constant & -1.235 & 0.285 & 18.814 & 1 & $<0.001$ & 0.291 & & \\
\hline
\end{tabular}

Hosmer-Lemeshow test $\chi^{2}(8)=7.165, p=0.519$. Abbreviations: CI, confidence interval; df, degrees of freedom. 


\section{Discussion}

This study brings new insights to the sleep field concerning both self-reported sleep quality and final awakening quality (SQ and AQ) in a large population study recruited from different regions of Portugal (mainland and islands) and from different subgroups, including sleep disorder patients.

The discussion involves the following aspects: (1) the used survey; (2) the SQ evaluation in the survey; (3) the use of AQ; (4) the prevalence values of SQ and AQ; (5) the associated factors and their functional meaning; (6) the risks of poor SQ and AQ.

The survey was constructed considering the following objectives: determination of individual and environmental factors influencing pandemic compliance, health, and sleep quality. Questions focused on standard demographic data, according to previous team surveys [42] or World Health Organization (WHO) and European Union (EU) question surveys or recommendations, as well as on clinical experience regarding schedules, habits, and attitudes. The existence of open questions allowed an enlarged scope in some respects. Most variables were either quantitative or dichotomic (yes/no). Sleep was only a chapter, whereby health evaluated both sleep and medical disorders. This differed from the International COVID-19 Sleep Study (ICOSS) survey [43], which focused on sleep/sleep disorders and used standard questionnaires; another major difference was the size: 50 questions for the ICOSS and 177 in our case. The ICOSS survey was international, while the present survey was focused on Portugal.

SQ evaluation is, in most surveys, done by using standard questionnaires, such as the PSQI [3], while AQ evaluation uses the SSA (Scale for Sleep and Awakening quality) questionnaire [8,9]. The different approach used by our group, via the application of VASs, was primarily to do with the extensive and multifocal questionnaire used and the need to reduce the number of questions as much as possible. Moreover, the questions relative to sleep schedules, sleep duration, and latencies implied both weekdays and weekends, prior to and during COVID-19. VASs achieve high response rates and high levels of completion, and there is significant evidence demonstrating their reliability in terms of inter-rater reliability and test-retest reliability; the differences observed between SQ and AQ favored the inexistence of response spreading, which is possible when using VASs [44]. A lack of precision, subjectiveness, and the frequent pessimistic attitude in evaluating complaints are common, especially in sleep disorder patients [45]. Altogether, these arguments led to a simple VAS with clear limits.

The use of an AQ scale reflects the recognition of the importance of the transition from sleep to awake, as discussed previously [8,9], together with its possible relevance for daytime consequences in terms of mood and behavior. Effectively, the AQ evaluation provided more frequent and consistent results than the SQ scale itself. This was shown where physical activity had a protective effect for poor AQ but not for SQ after adjusting for other variables such as age and gender. These results differ from those reported in other studies, where physical exercise practice is often associated with SQ improvement [46]. This relationship with AQ should be further studied since the AQ is also often dependent on SQ. In a recent study, the relationship between SQ of the previous night of sleep and a better cognitive performance (i.e., $\mathrm{AQ}$ performance) on the subsequent day was clearly shown for a sample of young adults [47].

The prevalence values of poor SQ during COVID-19, albeit high (SQ $=36.3 \%$ and AQ 34.6\%), are lower than those obtained by Italian authors $[13,14]$ but higher than those observed in China [15]; these differences are likely due to the differences in COVID-19 severity between countries and its consequent impact upon mood and perception [48] Compared with the pre-COVID-19 period, the average values of SQ and AQ were in fact significantly lower. However, it must be stated that, for SQ, $4.2 \%$ of respondents improved and $44.6 \%$ had no change; the corresponding values for AQ were $4.8 \%$ and $48.8 \%$, respectively. This means that deterioration was achieved by half of the population, whereas the other half remained equal or better; this shows a high resilience to stressing 
situations, a fact already reported in a recent study for a large population under the COVID-19 pandemic [49].

Our sample had a wide age distribution, and it is currently assumed that older age negatively impacts SQ. In this study, older people reported a significantly better AQ, while age differences for SQ were not found. This relationship with aging might be explained by the appearance of feeling refreshed after a night of sleep and easiness of awakening. In line with these results in the elderly, the wake drive predominates in relation to the weakening of the sleep drive [50].

In our study, women reported a significantly worse SQ and AQ than men. Poorer SQ in females was observed by others [51,52]; this is likely due to the specificities of sleep in the female gender [53]. Women tend to have a higher prevalence of poor sleep, independent of depression, sociodemographic, and lifestyle factors [51], and the difference is most likely a biological issue [53].

Both SQ and AQ are significantly associated with several factors. Like other studies, we found poor SQ and AQ in young adults [11], females [10,11,18,19], higher-education individuals [19,26], and healthcare workers (medical doctors (MDs) and nurses) $[7,14,24,31-33]$; however, civil status was not an influencing factor, except for $\mathrm{AQ}$, and the oldest group had higher SQ and AQ levels. The differences in married people are in line with the data concerning the number of people living together; SQ was better for two persons while $\mathrm{AQ}$ was better for 1-4 persons. Both SQ and AQ were poorer in larger households. Better results in the elderly might be related to a better acceptance of living circumstances, but we have no data to prove this.

Mood evaluation, the calamity scale, and facing economic problems were significantly associated with SQ and AQ. The data concerning anxiety, stress, depression [10,11,13-24], and worries [29] are in line with published results for SQ; the differences obtained concern the calamity scale and economic concerns. This might be due to the time of collection, since, in the present study, although undertaken during the first wave of COVID-19, data were collected until August, with people already facing 5 months of COVID-restrictions and direct and indirect economic impacts with, in many cases, job losses. The cited studies were performed at an earlier time point of the pandemic situation. This study highlights once more the relevance of SQ in mood and behavior especially for stressful situations [1,2].

The associations between SQ and other sleep variables were restricted to sleep latency and night waking on weekends and weekdays, as also established by others [10]. There was no association between SQ and sleep duration as conventionally assumed, although it existed for AQ. Both SQ and AQ were associated with get up time on weekdays, but in opposite directions. These discrepancies enhance the difficulties in the evaluation of subjective sleep data, reinforcing the need for objective data.

HRBs were significantly associated with AQ, specifically, physical activity $[19,23,54]$, while reinforcing the need for proper nutrition in terms of meals and food quality $[53,55]$. This enhances the need to moderate the use of social networks and mobile phones, and it highlights the dependence problem of modern technologies [23]. The timings of both exercise [56] and nutrition [57], although relevant for sleep, were not evaluated, due to the need to restrict the number of survey questions.

Attitudes and behaviors during COVID-19 were evaluated in detail in this study. Negative attitudes were associated with poor SQ [24,26,28] and AQ. Positive attitudes and behaviors were associated with good AQ; however, surprisingly, this also occurred for negative behaviors. The knowledge, attitudes, and practices that people hold toward the disease play an integral role in determining a society's readiness to accept behavioral change measures from health authorities in response to the COVID-19 pandemic, according to the study by Azlan et al. (2020) [58]. Data presented by Li et al. (2020) [59] corroborate these results, which showed that participants' knowledge about COVID-19 was positively related to social participation and precautionary behavior. However, when there was greater perceived severity of the situation, together with the perceived controllability as negative, there was an association with sleep problems and negative emotions. 
COVID19 infection, in a relatively small group, with many asymptomatic subjects, was significantly associated with poor SQ; the time delay between infection and the survey answer was, however, not collected. Available data show worse SQ in more severe patients [60].

Underweight was associated with poor AQ. Increased morbidities and a higher level of worsening morbidities were associated with poor SQ and AQ, even after adjusting for age and gender through the logistic model. However, improving morbidities had a clearcut association with good AQ, whereas the effect was surprisingly opposite with SQ. This apparently odd result might reflect the difficult evaluation of subjective $S Q$, which, linked to multiple components of sleep, such as latency, duration, and interruptions, is liable to subjective misperception $[45,61]$.

The self-reported cross-sectional nature of this report is a limitation that needs to be addressed. This study was not a national prevalence study and was based online, which can be considered a selection bias. Although the study did not include a national randomized sample, we consider it to be a representative study, since we had answers from all Portuguese regions, including the islands (Madeira and Azores). An aspect that also brought some strength to our study was the collection of many relevant variables that may impact daily behaviors, as well as affect the response to social restrictions, such as general health, marital status, children, and socioeconomic status.

\section{Conclusions}

This study provides a global view of what is recommended during COVID-19 or equivalent catastrophic periods. To sleep well, while having a high stress compliance, each individual should not only sleep but also have important control of their mood, practice positive behaviors, dismiss negative behaviors and attitudes, practice physical exercise, take care with meals and adequate nutrition, and beware of technologies and dependences.

Author Contributions: Conceptualization, T.P., A.F., T.G., and M.G.M.; Data curation, T.P., C.R., A.F., M.A.M., M.R.S., L.R., S.G., C.B. (Carla Bentes), F.S., L.P., C.P., A.C., S.M., I.L., J.P., G.V., J.F., A.B., J.V.C., S.R., and M.G.M.; formal analysis, T.P., C.R., T.G., G.T., and C.B. (Cátia Branquinho); investigation, T.P., C.R., A.F., H.C.-S., M.A.M., T.G., G.T., C.B. (Cátia Branquinho), M.R.S., L.R., S.G., C.B. (Carla Bentes), F.S., L.P., C.P., S.M., I.L., J.P., G.V., J.F., A.B., J.V.C., S.R., K.A., H.C., and M.G.M.; methodology, T.P., C.R., L.R., S.G., C.B. (Carla Bentes), F.S., L.P., C.P., S.M., I.L., J.P., G.V., J.F., A.B., K.A., H.C., and M.G.M.; resources, A.F. and M.R.S.; software, T.P.; supervision, T.P., K.A., H.C., and M.G.M.; writing—original draft, T.P., C.R., and M.G.M.; writing-review and editing, T.P., C.R., A.F., H.C.-S., M.A.M., T.G., G.T., C.B. (Cátia Branquinho), M.R.S., L.R., S.G., C.B. (Carla Bentes), F.S., L.P., C.P., A.C., S.M., I.L., J.P., G.V., J.F., A.B., J.V.C., S.R., K.A., H.C., and M.G.M. All authors have read and agreed to the published version of the manuscript.

Funding: This research did not receive any specific grant from funding agencies in the public, commercial, or nonprofit sections.

Institutional Review Board Statement: The project was approved by the Official Ethics Committee of CENC (Center of Sleep Medicine), Lisbon, Approval number 1/2020.

Informed Consent Statement: Informed consent was obtained before survey fulfillment.

Data Availability Statement: Data availability is controlled by the task leader, Prof Teresa Paiva; furthermore, the data will exclusively be used throughout at least 2021 by the "COVID, Health, Sleep, Habits" group, which includes, among others, the present authors. 
Acknowledgments: Gina Tomé is a postdoctorate funded by the Fundação Ciência e Tecnologia (FCT) SFRH/BPD/108637/2015. Aurora Lino' from the Lab. de Estudo do Sono, Hospital Santo Espírito da Ilha Terceira, Portugal, provided sleep disorder patients from Ilha Terceira, Azores. Rute de Sousa was an important liaison with the Psychologists Portuguese Association. We are thankful to the following professional associations for disseminating and promoting the surveys among their members:, the Portuguese Medical Association (Ordem dos Médicos), Portuguese Nurses Association (Ordem dos Enfermeiros), Portuguese Psychologists Association (Ordem dos Psicólogos), and Portuguese Pharmacists Association (Ordem dos Farmacêuticos). We thank the endorsement of the WASM (World Association of Sleep Medicine), ESRS (European Sleep Research Society), and APS (Associação Portuguesa de Sono).

Conflicts of Interest: The authors declare no conflict of interest. Linde-Saude, which includes a coauthor of this paper, paid the publication costs.

\section{Appendix A}

Criteria for Recommended Frequency of Food according to National directives [41]: "3 times/day", fruits/vegetables; milk and derivatives; carbohydrates/cereals; "1 or 2 times/day", dry fruits; legumes; tea/coffee; "2 times/day", meat/fish; "2 to 4 times week", eggs; "never", processed food; fast food; charcuterie; sweets and can sweets and candies; chocolates, sweet desserts; soft drinks.

\section{References}

1. Morin, C.M.; Carrier, J.; Bastien, C.; Godbout, R. Sleep and circadian rhythm in response to the COVID-19 pandemic. Canad. J. Public Health 2020, 111, 654-657. [CrossRef]

2. Kim, S.W.; Su, K.P. Using psychoneuroimmunity against COVID-19. Brain Behav. Immun. 2020, 87, 4-5. [CrossRef]

3. Mollayeva, T.; Thurairajah, P.; Burton, K.; Mollayeva, S.; Shapiro, C.M.; Colantonio, A. The Pittsburgh sleep quality index as a screening tool for sleep dysfunction in clinical and non-clinical samples: A systematic review and meta-analysis. Sleep Med. Rev. 2016, 25, 52-73. [CrossRef] [PubMed]

4. Jenkins, C.D.; Stanton, B.A.; Niemcryk, S.J.; Rose, R.M. A scale for the estimation of sleep problems in clinical research. J. Clin. Epidemiol. 1988, 41, 313-321. [CrossRef]

5. Hays, R.D.; Martin, S.A.; Sesti, A.M.; Spritzer, K.L. Psychometric properties of the Medical Outcomes Study Sleep measure. Sleep Med. 2005, 6, 41-44. [CrossRef]

6. Bastien, C. Validation of the Insomnia Severity Index as an outcome measure for insomnia research. Sleep Med. 2001, 2, 297-307. [CrossRef]

7. Paiva, T.; Penzel, T. Centro de Medicina do Sono: Manual Prático; Lidel: Lisboa, Portugal, 2011; ISBN 978-97-2757-719-4.

8. Saletu, B.; Wessely, P.; Grünberger, J.; Schultes, M. Erste klinische Erfahrungen mit einem neuen schlafanstoßenden Benzodiazepin, Cinolazepam, mittels eines Selbstbeurteilungsbogens für Schlaf-und Aufwachqualität (SSA). Neuropsychiatrie 1987, 1, 169-176.

9. Rosipal, R.; Lewandowski, A.; Dorffner, G. In search of objective components for sleep quality indexing in normal sleep. Biol. Psychol. 2013, 94, 210-220. [CrossRef] [PubMed]

10. Ohayon, M.; Wickwire, E.M.; Hirshkowitz, M.; Albert, S.M.; Avidan, A.; Daly, F.J.; Dauvilliers, Y.; Ferri, R.; Fung, C.; Gozal, D.; et al. National Sleep Foundation's sleep quality recommendations: First report. Sleep Health 2017, 3, 6-19. [CrossRef]

11. Trakada, A.; Nikolaidis, P.T.; Andrade, M.; Puccinelli, P.J.; Economou, N.T.; Steiropoulos, P.; Knechtle, B.; Trakada, G. Sleep During "Lockdown" in the COVID-19 Pandemic. Int. J. Environ. Res. Public Health 2020, 17, 9094. [CrossRef]

12. Zhou, Y.; Yang, Y.; Shi, T.; Song, Y.; Zhou, Y.; Zhang, Z.; Guo, Y.; Li, X.; Liu, Y.; Xu, G.; et al. Prevalence and Demographic Correlates of Poor Sleep Quality Among Frontline Health Professionals in Liaoning Province, China During the COVID-19 Outbreak. Front. Psychiatry 2020, 11. Available online: https://www.frontiersin.org/articles/10.3389/fpsyt.2020.00520/full (accessed on 28 December 2020). [CrossRef] [PubMed]

13. Franceschini, C.; Musetti, A.; Zenesini, C.; Palagini, L.; Scarpelli, S.; Quattropani, M.C.; Lenzo, V.; Freda, M.F.; Lemmo, D.; Vegni, E.; et al. Poor Sleep Quality and Its Consequences on Mental Health During the COVID-19 Lockdown in Italy. Front. Psychol. 2020, 11. Available online: https://www.frontiersin.org/articles/10.3389/fpsyg.2020.574475/full?\&utm_ source=Email_to_authors_\&utm_medium=Email\&utm_content=T1_11.5e1_author\&utm_campaign=Email_publication\& field=\&journalName=Frontiers_in_Psychology\&id=574475 (accessed on 28 December 2020). [CrossRef]

14. Casagrande, M.; Favieri, F.; Tambelli, R.; Forte, G. The enemy who sealed the world: Effects quarantine due to the COVID-19 on sleep quality, anxiety, and psychological distress in the Italian population. Sleep Med. 2020, 75, 12-20. [CrossRef] [PubMed]

15. Wang, X.; Lei, S.M.; Le, S.; Yang, Y.; Zhang, B.; Yao, W.; Gao, Z.; Cheng, S. Bidirectional Influence of the COVID-19 Pandemic Lockdowns on Health Behaviors and Quality of Life among Chinese Adults. Int. J. Environ. Res. Public Health 2020, 17, 5575. [CrossRef]

16. Florea, C.; Topalidis, P.; Hauser, T.; Angerer, M.; Kurapov, A.; Beltran Leon, C.A.; Soares Brandão, D.; Schabus, M. Sleep during COVID-19 lockdown: A cross-cultural study investigating job system relevance. Biochem. Pharmacol. 2021, 10, 114463. [CrossRef] 
17. Reis, C.; Dias, S.; Rodrigues, A.M.; Sousa, R.D.; Gregório, M.J.; Branco, J.; Canhão, H.; Paiva, T. Sleep duration, lifestyles and chronic diseases: A cross-sectional population-based study. Sleep Sci. 2018, 11, 217-230. [CrossRef] [PubMed]

18. Xiao, H.; Zhang, Y.; Kong, D.; Li, S.; Yang, N. Social Capital and Sleep Quality in Individuals Who Self-Isolated for 14 Days During the Coronavirus Disease 2019 (COVID-19) Outbreak in January 2020 in China. Med. Sci. Monit. 2020, 26. Available online: https:/ / www.ncbi.nlm.nih.gov/pmc/articles/PMC7177038/ (accessed on 28 December 2020). [CrossRef]

19. Huang, Y.; Zhao, N. Generalized anxiety disorder, depressive symptoms and sleep quality during COVID-19 outbreak in China: A web-based cross-sectional survey. Psychiatry Res. 2020, 288, 112954. [CrossRef]

20. Idrissi, J.A.; Lamkaddem, A.; Benouajjit, A.; ben El Bouaazzaoui, M.; el Houari, F.; Alami, M.; Labyad, S.; Chahidi, A.; Benjelloun, M.; Rabhi, S.; et al. Sleep quality and mental health in the context of COVID-19 pandemic and lockdown in Morocco. Sleep Med. 2020, 74, 248-253. [CrossRef]

21. Marelli, S.; Castelnuovo, A.; Somma, A.; Castronovo, V.; Mombelli, S.; Bottoni, D.; Leitner, C.; Fossati, A.; Ferini-Strambi, L. Impact of COVID-19 lockdown on sleep quality in university students and administration staff. J. Neurol. 2020. Available online: https: / / link.springer.com/article/10.1007/s00415-020-10056-6 (accessed on 20 December 2020). [CrossRef]

22. Fu, W.; Wang, C.; Zou, L.; Guo, Y.; Lu, Z.; Yan, S.; Mao, J. Psychological health, sleep quality, and coping styles to stress facing the COVID-19 in Wuhan, China. Transl. Psychiatry 2020, 10, 225. [CrossRef]

23. Bigalke, J.A.; Greenlund, I.M.; Carter, J.R. Sex differences in self-report anxiety and sleep quality during COVID-19 stay-at-home orders. Biol. Sex. Differ. 2020, 11, 56. [CrossRef]

24. Yang, Y.; Zhu, J.; Yang, S.; Lin, H.; Chen, Y.; Zhao, Q.; Fu, C. Prevalence and associated factors of poor sleep quality among Chinese returning workers during the COVID-19 pandemic. Sleep Med. 2020, 73, 47-52. [CrossRef]

25. Korkmaz, S.; Kazgan, A.; Çekiç, S.; Tartar, A.S.; Balcı, H.N.; Atmaca, M. The anxiety levels, quality of sleep and life and problem-solving skills in healthcare workers employed in COVID-19 services. J. Clin Neurosci 2020, 80, 131-136. [CrossRef] [PubMed]

26. Werneck, A.O.; Silva, D.R.; Malta, D.C.; Lima, M.G.; Souza-Júnior, P.R.B.; Azevedo, L.O.; Barros, M.B.A.; Szwarcwald, C.L. The mediation role of sleep quality in the association between the incidence of unhealthy movement behaviors during the COVID-19 quarantine and mental health. Sleep Med. 2020, 76, 10-15. [CrossRef] [PubMed]

27. Wang, W.; Song, W.; Xia, Z.; He, Y.; Tang, L.; Hou, J.; Lei, S. Sleep Disturbance and Psychological Profiles of Medical Staff and Non-Medical Staff During the Early Outbreak of COVID-19 in Hubei Province, China. Front. Psychiatry 2020, 11. Available online: https:/ / www.frontiersin.org/articles/10.3389/fpsyt.2020.00733/full (accessed on 31 December 2020). [CrossRef] [PubMed]

28. Gupta, R.; Grover, S.; Basu, A.; Krishnan, V.; Tripathi, A.; Subramanyam, A.; Nischal, A.; Hussain, A.; Mehra, A.; Ambekar, A.; et al. Changes in sleep pattern and sleep quality during COVID-19 lockdown. Indian J. Psychiatry 2020, 62, 370. [CrossRef]

29. Peltz, J.S.; Daks, J.S.; Rogge, R.D. Mediators of the association between COVID-19-related stressors and parents' psychological flexibility and inflexibility: The roles of perceived sleep quality and energy. J. Contextual. Behav. Sci. 2020, 17, 168-176. [CrossRef] [PubMed]

30. Ingram, J.; Maciejewski, G.; Hand, C.J. Changes in Diet, Sleep, and Physical Activity Are Associated With Differences in Negative Mood during COVID-19 Lockdown. Front. Psychol. 2020, 11. [CrossRef]

31. Targa, A.D.S.; Benítez, I.D.; Moncusí-Moix, A.; Arguimbau, M.; de Batlle, J.; Dalmases, M.; Barbé, F. Decrease in sleep quality during COVID-19 outbreak. Sleep Breath 2020. Available online: https:/ /link.springer.com/article/10.1007/s11325-020-02202-1 (accessed on 31 December 2020). [CrossRef]

32. Grossman, E.S.; Hoffman, Y.S.G.; Palgi, Y.; Shrira, A. COVID-19 related loneliness and sleep problems in older adults: Worries and resilience as potential moderators. Pers. Individ. Dif. 2021, 168, 110371. [CrossRef]

33. Martínez-Lezaun, I.; Santamaría-Vázquez, M.; del Líbano, M. Influence of Confinement by COVID-19 on the Quality of Sleep and the Interests of University Students. Nat. Sci. Sleep 2020, 12, 1075-1081. [CrossRef] [PubMed]

34. Jahrami, H.; BaHammam, A.S.; AlGahtani, H.; Ebrahim, A.; Faris, M.; AlEid, K.; Saif, Z.; Haji, E.; Dhahi, A.; Marzooq, H.; et al. The examination of sleep quality for frontline healthcare workers during the outbreak of COVID-19. Sleep Breath 2020. [CrossRef]

35. Stojanov, J.; Malobabic, M.; Stanojevic, G.; Stevic, M.; Milosevic, V.; Stojanov, A. Quality of sleep and health-related quality of life among health care professionals treating patients with coronavirus disease-19. Int. J. Soc. Psychiatry 2020, 002076402094280. Available online: https: / journals.sagepub.com/doi/full/10.1177/0020764020942800 (accessed on 31 December 2020). [CrossRef] [PubMed]

36. Qi, J.; Xu, J.; Li, B.; Huang, J.; Yang, Y.; Zhang, Z.; Yao, D.; Liu, Q.; Jia, M.; Gong, D.; et al. The evaluation of sleep disturbances for Chinese frontline medical workers under the outbreak of COVID-19. Sleep Med. 2020, 72, 1-4. [CrossRef] [PubMed]

37. Barrea, L.; Pugliese, G.; Framondi, L.; di Matteo, R.; Laudisio, D.; Savastano, S.; Colao, A.; Muscogiuri, G. Does Sars-Cov-2 threaten our dreams? Effect of quarantine on sleep quality and body mass index. J. Transl. Med. 2020, 18, 318. [CrossRef]

38. Kocevska, D.; Blanken, T.F.; van Someren, E.J.W.; Rösler, L. Sleep quality during the COVID-19 pandemic: Not one size fits all. Sleep Med. 2020, 76, 86-88. [CrossRef]

39. Ohayon, M.M. Prevalence and Risk Factors of Morning Headaches in the General Population. Arch. Intern. Med. 2004, 164-197. [CrossRef]

40. Ohayon, M.M.; Dauvilliers, Y.; Milesi, C. Définitions et épidémiologie de L’hypersomnolence. La plainte "somnolence excessive" concerne un quart de la population. La Revue Du Prat. 2016, 66, 654-659. 
41. Gregório, M.J.; Santos, C.T.; Ferreira, S.; Graça, P. Alimentação Inteligente-Coma Melhor, Poupe Mais; Direção-Geral da Saúde: Lisboa, Portugal, 2012; pp. 6-7. ISBN 978-972-675-197-7.

42. De Matos, M.G.; Barrett, P.; Dadds, M.; Shortt, A. Anxiety, depression, and peer relationships during adolescence: Results from the Portuguese national MG health behaviour in school-aged children survey. Eur. J. Psychol. Educ. 2003, 18, 3-14. [CrossRef]

43. Partinen, M.; Bjorvatn, B.; Holzinger, B.; Chung, F.; Penzel, T.; Espie, C.A.; Morin, C.M. ICOSS-collaboration group. Sleep and circadian problems during the coronavirus disease 2019 (COVID-19) pandemic: The International COVID-19 Sleep Study (ICOSS). J Sleep Res. 2021, 30, e13206. [CrossRef]

44. Brazier, J.; Ratcliffe, J. Measurement and Valuation of Health for Economic Evaluation. In International Encyclopedia of Public Health, 2nd ed.; Quah Stella, R., Ed.; Academic Press: Cambridge, MA, USA, 2017; pp. 586-593. ISBN 9780128037089. [CrossRef]

45. Mestre, C.; Rebelo Pinto, T.; Rebelo Pinto, H.; Paiva, T. Errors in sleep duration perception in insomniac patient. J. Sleep Res. 2016, 25,308 .

46. Banno, M.; Harada, Y.; Taniguchi, M.; Tobita, R.; Tsujimoto, H.; Tsujimoto, Y.; Kataoka, Y.; Noda, A. Exercise can improve sleep quality: A systematic review and meta-analysis. Peer] 2018, 6, e5172. [CrossRef] [PubMed]

47. Zavecz, Z.; Nagy, T.; Galkó, A.; Nemeth, D.; Janacsek, K. The relationship between subjective sleep quality and cognitive performance in healthy young adults: Evidence from three empirical studies. Sci. Rep. 2020, 10, 4855. [CrossRef] [PubMed]

48. Ben Salah, A.; DeAngelis, B.N.; al'Absi, M. Resilience and the Role of Depressed and Anxious Mood in the Relationship between Perceived Social Isolation and Perceived Sleep Quality During the COVID-19 Pandemic. Int. J. Behav. Med. 2021, 7, 1-9. [CrossRef]

49. Barzilay, R.; Moore, T.M.; Greenberg, D.M.; DiDomenico, G.E.; Brown, L.A.; White, L.K.; Gur, R.C.; Gur, R.E. Resilience, COVID-19-related stress, anxiety and depression during the pandemic in a large population enriched for healthcare providers. Transl. Psychiatry 2020, 10, 291. [CrossRef] [PubMed]

50. Putilov, A.A. Age-related changes in the association of sleep satisfaction with sleep quality and sleep-wake pattern Sleep Biol. Rhythms. 2017. Available online: https://link.springer.com/article/10.1007/s41105-017-0140-8 (accessed on 30 December 2020). [CrossRef]

51. Fatima, Y.; Doi, S.A.R.; Najman, J.M.; Mamun, A. Exploring Gender Difference in Sleep Quality of Young Adults: Findings from a Large Population Study. Clin. Med. Res. 2016, 14, 138-144. [CrossRef]

52. Tang, J.; Liao, Y.; Kelly, B.C.; Xie, L.; Xiang, Y.; Qi, C.; Pan, C.; Hao, W.; Liu, T.; Zhang, F.; et al. Gender and Regional Differences in Sleep Quality and Insomnia: A General Population-based Study in Hunan Province of China. Sci. Rep. 2017, 7, 43690. [CrossRef] [PubMed]

53. Mallampalli, M.P.; Carter, C.L. Exploring Sex and Gender Differences in Sleep Health: A Society for Women's Health Research Report. J. Womens Health 2014, 23, 553-562. [CrossRef]

54. Robinson, E.; Boyland, E.; Chisholm, A.; Harrold, J.; Maloney, N.G.; Marty, L.; Mead, B.R.; Noonan, R.; Hardman, C.A. Obesity, eating behavior and physical activity during COVID-19 lockdown: A study of UK adults. Appetite 2021, 156, 104853. [CrossRef]

55. Poelman, M.P.; Gillebaart, M.; Schlinkert, C.; Dijkstra, S.C.; Derksen, E.; Mensink, F.; Hermans, R.C.J.; Ardening, P.; de Ridder, D.; de Vet, E. Eating behavior and food purchases during the COVID-19 lockdown: A cross-sectional study among adults in the Netherlands. Appetite 2021, 157, 105002. [CrossRef]

56. Dolezal, B.A.; Neufeld, E.V.; Boland, D.M.; Martin, J.L.; Cooper, C.B. Interrelationship between Sleep and Exercise: A Systematic Review. Adv. Prev Med. 2017, 2017, 1364387. [CrossRef]

57. Culnan, E.; Reid, K.J.; Zee, P.C.; Crowley, S.J.; Baron, K.G. Meal timing relative to DLMO: Associations with BMI and body fat. Sleep Health. 2021, 17. Available online: https://www.sciencedirect.com/science/article/abs/pii/S2352721821000012 (accessed on 31 December 2020). [CrossRef]

58. Azlan, A.A.; Hamzah, M.R.; Sern, T.J.; Ayub, S.H.; Mohamad, E. Public knowledge, attitudes and practices towards COVID-19: A cross-sectional study in Malaysia. PLoS ONE 2020, 15, e0233668. [CrossRef]

59. Li, J.; Yang, A.; Dou, K.; Wang, L.; Zhang, M.; Lin, X. Chinese public's knowledge, perceived severity, and perceived controllability of COVID-19 and their associations with emotional and behavioural reactions, social participation, and precautionary behaviour: A national survey. BMC Public Health 2020, 20, 1589. [CrossRef] [PubMed]

60. Vitale, J.A.; Perazzo, P.; Silingardi, M.; Biffi, M.; Banfi, G.; Negrini, F. Is disruption of sleep quality a consequence of severe Covid-19 infection? A case-series examination. Chronobiol. Int. 2020, 37, 1110-1114. [CrossRef] [PubMed]

61. Trimmel, K.; Eder, H.G.; Böck, M.; Stefanic-Kejik, A.; Klösch, G.; Seidel, S. The (mis)perception of sleep: Factors influencing the discrepancy between self-reported and objective sleep parameters. J. Clin. Sleep Med. 2021, 33393901. Available online: https:/ /jcsm.aasm.org/doi/10.5664/jcsm.9086 (accessed on 30 December 2020). [CrossRef] 